\title{
Life-review therapy with computer supplements for depression in the elderly: A randomized controlled trial
}

Barbara Preschl, M.A. ${ }^{1 \S}$, Andreas Maercker, Ph.D. ${ }^{1}$, Birgit Wagner, Ph.D. ${ }^{2}$, Simon Forstmeier, Ph.D. ${ }^{1}$, Rosa M. Baños, Ph.D. ${ }^{3}$, Mariano Alcañiz, Ph.D. ${ }^{4}$, Diana Castilla, B.A. ${ }^{5}$, Cristina Botella, Ph.D. ${ }^{5}$

${ }^{1}$ Department of Psychopathology and Clinical Intervention, University of Zurich, Zurich, Switzerland

${ }^{2}$ Clinic for Psychotherapy and Psychosomatic Medicine, University Hospital Leipzig, Leipzig, Germany

${ }^{3}$ Departamento Personalidad, Evaluación y Tratamientos Psicológicos, Universidad de Valencia, Spain

${ }^{4}$ LabHuman, Universidad Politécnica de Valencia, Valencia, Spain

${ }^{5}$ Departamento de Psicología Básica, Universitat Jaume I, Castellón, Spain

${ }^{\S}$ Corresponding author

Barbara Preschl

Department of Psychopathology and Clinical Intervention, University of Zurich Binzmühlestrasse 14/17

8050 ZürichSwitzerlandTel.: +41 446357458

E-Mail: b.preschl@psychologie.uzh.ch 
Abstract

Objectives

Life-review therapy has been recognized as an effective therapeutic approach for depression in older adults. Additionally, the use of new media is becoming increasingly common in psychological interventions. The aim of this study was to investigate a life-review therapy in a face-to-face setting with additional computer use.

\section{Method}

This study explored whether a six-week life-review therapy with computer supplements from the e-mental health Butler system constitutes an effective approach to treat depression in older adults aged 65 and over. A total of 36 participants with elevated levels of depressive symptoms were randomized to a treatment group or a waiting-list control group and completed the postassessment. 14 individuals in the intervention group completed the follow-up assessment.

Results

Analyses revealed significant changes from pre- to post-treatment or follow-up for depression, well-being, self-esteem and obsessive reminiscence, but not for integrative reminiscence and life-satisfaction. Depressive symptoms decreased significantly over time until the 3-month follow-up in the intervention group compared to the control group (pre to post: $d=1.13$; pre to follow-up: $d=1.27$; group x time effect pre to post: $d=0.72$ ). Furthermore, the therapy led to an increase in well-being and a decrease in obsessive reminiscence among the participants in the intervention group from pre-treatment to follow-up (wellbeing: $d=.70$; obsessive reminiscence: $d=.93$ ). Analyses further revealed a significant but small group $\mathrm{x}$ time effect regarding self-esteem $(d=.19)$.

\section{Conclusion}

By and large, the results indicate that the life-review therapy in this combined setting could be recommended for depressive older adults.

Keywords: depression, life-review, e-mental health, older adults 


\section{Introduction}

Since unipolar depression is among the most frequent mental disorders in old age (Wernicke, Linden, Gilberg, \& Helmchen, 2000), the development of new psychotherapeutic methods or the adaptation of existing ones is highly relevant. Results from the Berlin Aging study (Wernicke, Linden, Gilberg, \& Helmchen, 2000), showed the following prevalence rates for unipolar depression in older adults aged 70 or above: unipolar depression (not otherwise specified, without major depression): 17.8\%, major depression (moderate, without psychotic symptoms): 4.2\%, major depression (severe, without psychotic symptoms): $0.5 \%$ ). As shown in the Berlin Aging study and demonstrated by others (Cole \& Dendukuri, 2003), the prevalence rates for mild to moderate depressive symptoms (unipolar) or subthreshold depression in old age are higher than for major depressive disorder while subthreshold depression is considered as a risk factor for developing a major depressive disorder (Beekman, et al., 2002).

In the current study, a life-review therapy with computer supplements for milder forms of depression in the elderly was investigated. The intervention consisted of two modules: a face-to-face life review part focusing on positive and negative past events; and a computer part (see below: "Butler" system, Botella, et al., 2009) focusing exclusively on positive experiences.

Webster, Bohlmeijer and Westerhof (2010) and Westerhof, Bohlmeijer and Webster (2010) have proposed distinctions between different forms of reminiscence interventions. The authors distinguish simple reminiscence (unstructured), life-review (more structured and integrative, focusing on the whole life-span) and life-review therapy (adopting life-review for the treatment of mental disorders). In the current study we conducted a life-review therapy for the treatment of depression. 
Life-review therapy focuses on the balance of positive and negative reminiscence (in terms of emotional valence), the redefinition of negative experiences and elaboration of memory (Maercker, 2002). Through structured questions, life-review therapy enables the individual to focus equally on positive and negative past events, with the aim of obtaining a coherent and balanced view of one's past life. Traditionally, different types of reminiscence have been distinguished (Webster, 1993, for more recent reviews see Webster, Bohlmeijer \& Westerhof, 2010; and Westerhof, Bohlmeijer \& Webster, 2010). Wong and Watt (1991) showed that some reminiscence types are more strongly related to successful aging (increased self-understanding, personal meaning, self-esteem and life satisfaction, p.273) than others, e.g., the integrative type, i.e. an achievement of a "sense of self-worth, coherence, and reconciliation with regard to one's past" (p.273) being positively related to well-being. Based on these findings, we aimed to investigate two different reminiscence types, integrative and obsessive reminiscence (i.e. a failure to integrate problematic past experiences, p. 273); the latter being related to less successful aging as compared to integrative reminiscence, which was associated with successful aging in the study by Wong and Watt (1991). Based on Wong and Watt's (1991) taxonomy Mayer, Filipp, and Ferring (1996) developed the first German reminiscence questionnaire that was used in the current study.

In a meta-analysis regarding the effectiveness of life review on late-life depression, Bohlmeijer, Smit, \& Cuijpers (2003) reported a large effect size $(d=0.84)$. Following Pinquart, Duberstein and Lyness (2007), effect sizes in that context drop when an active control group is involved. Recently, Pinquart and Forstmeier (2012) reported a medium effect size for depression at post-treatment $(g=0.57)$. The effect size increased for depressed individuals who received life-review therapy $(g=1.28)$. 
Based on the fact that depressed individuals as well as older adults are often unable to recall specific events, i.e. recall more general ("over general") memories, Serrano, Latorre, Gatz, and Montanes (2004) investigated a life-review therapy based on a training of the retrieval of specific positive memories. The authors showed in a randomized controlled trial that the integration of "autobiographical retrieval practice" into life review, focusing mainly on memories for positive events, proved to be an effective intervention tool for depressive older adults aged 65 or over. Based on these findings, a module was integrated into the current study focusing on positive events in a computer-guided setting (see below).

Besides depressive symptoms, life review therapy has also shown positive effects on self-esteem (in terms of self-respect, to consider oneself a person of worth; Gray-Little, Williams \& Hancock, 1997, following Rosenberg 1979). Chiang, Lu, Chu, Chang, and Chou (2008) showed in a randomized controlled trial that a "Life-Review Group Program" (LRGP) positively influenced the self-esteem of elderly males (mean age 78.13 years). In an earlier study, Haight and Dias (1992) investigated the effects of ten different forms of reminiscence on depression and self-esteem. Results showed that a structured (covering life from birth to present, p. 282) and evaluative form (evaluation of feelings, p.282) of life review is more effective than other forms with regard to depression and self-esteem. In a recent meta-analysis Pinquart and Forstmeier (2012) reported small effect sizes regarding self-esteem at post-treatment $(g=0.20)$.

Furthermore, life-review therapy has shown positive effects on satisfaction with life and/or well-being (Bohlmeijer, Roemer, Cuijpers, \& Smit, 2007; Chiang, et al., 2008). Bohleijer et al. (2007) reported a medium effect size $(d=0.54)$ in this context, whereas Pinquart and Forstmeier (2012) reported small effect sizes regarding lifesatisfaction $(g=0.22)$ and positive well-being $(g=0.33)$ at post-treatment. Based on 
these findings, in the current study, we further investigated the effects of our intervention on life satisfaction (comprising 5 dimensions: zest versus apathy, resolution and fortitude, congruence between desired and achieved goals, positive self-concept, and mood tone; Liang, 1984, following Neugarten et al., 1961) and general well-being (positive mood, vitality, interest in things; Bech, 1998).

As mentioned above, the life-review therapy conducted in the current study also contained a computer intervention part, comprising two 'depression modules' of a computer program ("Butler" system, Botella, et al., 2009) (as detailed below). In general, e-health interventions targeting older adults have been recognized as a promising approach for a variety of domains including depression, although research in this field is still in its infancy (Preschl, Wagner, Forstmeier, \& Maercker, 2011). Moreover, therapeutic software has been used successfully as a supplement in traditional face-to-face therapy, e.g. "Virtual Reality Exposure Therapy" (Parsons \& Rizzo, 2008), in which a computer tool (a simulation or virtual environment) can be used in a therapeutic setting for the treatment of anxiety and specific phobias. With regard to depression (and anxiety), a computer program containing several modules was developed by a Spanish research team, the so-called "Butler system" (Spanish: "Mayordomo") (Botella, et al., 2009). This system provides various fields of application for older adults: diagnosis and therapy (depression and anxiety) and social interaction modules (also for healthy older adults). In the current study, two therapy modules of this system for treating depression were used, which provide the possibility to focus on certain events in the context of life review and autobiographical memory. The first module contains so-called "Virtual Environments" (VE), in which the user learns techniques to reduce negative mood and to recall and describe positive autobiographical memories. This module provides three exercises: a mindfulness-based intervention 
focusing on the recognition of one-self in the actual situation in preparation of the further exercises, a relaxation exercise for agitated depressed individuals and a guided exercise focusing on the recall of positively valuated episodes in one's life. The second module, a 3D adaptation of a book containing several chapters, is called the "Book of Life". By incorporating text, pictures, and Mp3 music files, the "Book of Life" can be customized by the user. This guided tool offers several possibilities to recall and deal with positive valuated episodes in one`s life. The "Butler system" contains touch screen technology and was developed and tested to meet the needs of older adults (Botella, et al., 2009). All applications are guided by a personalized icon, the so-called "butler", or in the case of the VE by a female voice, which describes all exercises to the user step by step. The "Butler system" was translated into German at the University of Zurich in cooperation with the aforementioned Spanish team, who integrated the German audio and text files into the system. The methods section of this article provides more information about how the depression modules of the "Butler system" were used in the current study.

Based on these findings, we conducted a randomized controlled study investigating a structured and time-limited (six-week) life-review therapy with depressive older adults in a face-to-face setting with additional use of the aforementioned depression modules of the "Butler system" (Botella, et al., 2009). To our knowledge, this is the first randomized controlled trial to investigate a life-review therapy in a combined e-mental health setting. The first objective of this study was to investigate whether this combined and short-term life-review therapy leads to a reduction in depressive symptoms. Second, we examined whether the intervention leads to an increase in self-esteem, life satisfaction and well-being. Third, we investigated two types of reminiscence: integrative and obsessive reminiscence. 


\section{Methods}

\section{Study design}

A randomized controlled trial comparing a face-to-face life-review therapy including computer supplements with a waiting-list control group was conducted at the University of Zurich. Both groups received a six-week intervention; for ethical reasons, the waiting-list control group received the same intervention after the waiting time period. Assessments were conducted at baseline and at post-treatment, and participants in the intervention group also participated in a 3-month follow-up session. The participants completed the Beck Depression Inventory (BDI-II, Hautzinger, Keller, \& Kühner (2006), the Rosenberg Self-Esteem Scale (SES, (Wendt, 1979), the Life Satisfaction Index - A (LSIA, (Wiendieck, 1970)), the Reminiscence Questionnaire (RQ, Mayer, Filipp, \& Ferring, 1996b), and the WHO-Five Well-being Index (WHO-5, Bech, 1998).

\section{Participants}

Participants were recruited between December 2009 and April 2011. The ethics committee of the German Psychological Society (DGPs) approved the study in December 2009. Patients were recruited through advertisements in newspapers, supermarkets, libraries, pharmacies, general practitioners' practices, a contact list of individuals who were generally interested in participating in research projects, and lectures for older adults at the University of Zurich. The contact list was prepared by a coordinator who administered spontaneous requests from individuals who were interested in participating in a research project or from former study participants who were interested in further participating in research projects.

Older adults aged 65 or over who suffered from minimal (subsyndromal) to moderate depression (BDI-II score 10-28) were included in the study. Exclusion criteria were cognitive impairment (MMSE, Folstein, Folstein, \& McHugh (1975) score 
below 27), severe depression (BDI-II score above 28, SKID, (Wittchen, Zaudig, \& Fydrich, 1997)), severe vision or hearing impairment (NAB, Oswald \& Fleischmann, 1995), mobility problems (unable to come to the outpatient clinic), currently receiving psychotherapeutic treatment elsewhere (during the treatment or waiting time period, but also between post-assessment and follow-up), indications of severe suicidal ideation (BDI-II, SKID), or other psychiatric disorders (SKID). The baseline assessment was conducted by the study coordinator (MA in psychology and CBT training). Study participants were asked to fill in all further questionnaires on their own (at home or at the University of Zurich in case they needed support). Master students were further involved in the assessments (e.g. giving instructions to the participants). Follow-up questionnaires were sent by mail.

Demographic characteristics of the sample are presented in Table 1. No significant differences in baseline characteristics were noted between groups, besides age $\left[\chi^{2}(1)=5.36, p<.05\right]$. The age difference was in the direction of the intervention group being older than the control group. The scores of the outcome measures of the two groups did not differ significantly at baseline, with the exception of life satisfaction (LSIA) $[\mathrm{t}(34)=-2.18, p<.05]$. Life satisfaction was lower at baseline for the intervention group compared to the control group

\section{Study Procedure}

Participants indicated their interest in the study by contacting the study coordinator via telephone or E-Mail. In this context, the study coordinator asked about basic characteristics (age, mobility, currently receiving psychotherapy) and provided general information concerning the procedure of the study and depressive symptoms. Subsequently, a meeting was arranged to give further information and check for inclusion and exclusion criteria. If the inclusion criteria were met, participants were 
randomly assigned to either the intervention group or a waiting-list control group at the end of the first meeting. The study coordinator used a true random number service (http://www.random.org) to organize the randomization procedure, which was not stratified by any participant characteristics. All participants were provided with detailed information regarding their participation in the intervention or control group (e.g. assessment time points, therapeutic procedure, or waiting time period for control group participants). They were further informed about potential risks and benefits of study participation and told that they could withdraw from the study at any time. All information was provided in oral and written form. In addition, participants signed an informed consent form. One week after the first meeting, the second meeting was arranged to assess the baseline data. Participants were encouraged to telephone or email the therapist during their study participation in the case of distress or crisis. Applicants excluded from the study were informed about other available forms of counseling or treatment. Participants in the intervention group began with the six-week intervention one week after the baseline assessment. For ethical reasons, the control group also received the same therapy after a six-week waiting time period.

Figure 1 shows a flow chart of participants. Twenty individuals in the intervention group and sixteen in the control group completed the post measurement. Furthermore, fourteen participants in the intervention group completed the follow-up. After beginning treatment, nobody dropped out before finishing the post-measurement, but six failed to complete the three-month follow-up. The main reasons given for discontinuing participation were lack of time, lack of motivation, transportation problems, or severe illness. Participants who dropped out before starting treatment or finishing baseline assessments were not considered in the analyses.

\section{Measures}




\section{Outcome measures}

Depression. Severity of depressive symptoms was measured using the German version (Hautzinger, et al., 2006) of the Beck Depression Inventory-II (BDI, Beck, Steer, \& Brown, 1996), a self-rating questionnaire with 21 items assessing specific symptoms of depression. The internal consistency in the current sample was $\alpha=.86$.

Self-esteem. Self-esteem (in terms of self-respect, to consider oneself a person of worth; Gray-Little, Williams \& Hancock, 1997) was assessed using the Rosenberg Self-Esteem Scale (RSES, Wendt, 1979), a 10-item scale (e.g. I feel that I have a number of good qualities. I feel I do not have much to be proud of). The internal consistency in the current sample was $\alpha=.70$.

Life Satisfaction. To assess life satisfaction, the Life Satisfaction Index A (LSIA, Wiendieck, 1970) was used. The LSIA is an 18-item self-report scale to measure life satisfaction especially in old age (e.g. I feel old and somewhat tired. My life could be happier than it is now). This scale comprises five dimensions: zest versus apathy, resolution and fortitude, congruence between desired and achieved goals, positive selfconcept, and mood tone (Liang, 1984, following Neugarten et al. 1961). The internal consistency in the current sample was $\alpha=.76$.

Well-being. General well-being (positive mood, vitality, interest in things) was measured using the WHO-Five Well-being Index (WHO-5, Bech, 1998), a five-item questionnaire (e.g. Over the last two weeks, I have felt calm and relaxed. Over the last two weeks I woke up fresh and rested). The internal consistency in the current sample was $\alpha=.89$.

Reminiscence Types. In the current study, integrative (3 items, e.g. I feel that even bad times were meaningful in my life.) and obsessive reminiscence (4 items, e.g. When reflecting on my past life, I very often feel guilty.) was assessed using the Reminiscence 
Questionnaire (RQ, Mayer, Filipp, \& Ferring, 1996a), a German questionnaire that was developed following the taxonomy of Wong and Watt (1991). Obsessive reminiscence is an equivalent to the type "bitterness revival" described by, Webster (1993). The internal consistency in the current sample was $\alpha=.64$ for integrative reminiscence, and $\alpha=.72$ for obsessive reminiscence.

\section{Exclusion criteria}

Cognitive impairment / dementia. Cognitive functioning was assessed by the Mini Mental State Examination (MMSE, Folstein, et al., 1975). Individuals who scored below 27 were excluded.

Suicidal ideation. Severe suicidal ideation was screened with the BDI-II and the Structured Clinical Interview for DSM-IV (SKID, Wittchen, et al., 1997) . Individuals who were excluded due to severe suicidal ideation were provided with support in addition to the general information for excluded individuals, e.g. informing significant others or other professionals (e.g. their general practitioner).

Other psychiatric disorders. To screen for other psychiatric disorders, we used the SKID (Wittchen, et al., 1997). Diagnoses were validated by experienced clinicians who were trained in structured clinical assessment.

Vision and hearing. Vision and hearing impairment was assessed using items 8 and 10 of the "Nuremberg Gerentopsychological Observation Scale" (NAB), an observer-rated subscale of the Nuremberg Gerentopsychological Inventory (NAI, Oswald \& Fleischmann, 1995). A score below 3 was considered as meeting the inclusion criteria.

\section{Therapists}

One male (Ph.D) and one female (M.A.) psychologist with training in psychotherapy and cognitive behavioral therapy (CBT) participated in this study. Both therapists were 
given special training in life-review therapy and in the application of the computer modules for this study including an introduction session by the project leader concerning the method itself (life-review therapy), a discussion of the treatment manual and of lessons learned from a case study (approximately 5 hours). Further the developers of the Butler system provided a detailed introduction of the system and further presented results from a case study (approximately 5 hours). The therapists received regular supervision by the project leader and further by external supervisors. Therapists were allocated to patients based on time and availability.

\section{Treatment}

In the context of the current study, a structured treatment manual was compiled following Haight and Haight (2007), Maercker (2002) and Serrano et al. (2004). In total, eight meetings were arranged with each participant. During the first two meetings (one meeting each in the first and second week), the participants answered questionnaires screening for inclusion criteria and answered the baseline assessment. In the third week, the intervention group began the treatment, meeting for one session per week for six weeks. Each session was divided into two parts: a face-to-face part (about two thirds of the session time) and a computer part (about one third of the session time). Each session lasted between 1 and 1.5 hours. In the first session, the patient was provided with a list of questions focusing on negative and positive experiences of his or her past life from childhood until old age covering the six sessions. The therapist provided questions focusing on the computer intervention and on the face-to-face intervention as shown in the following examples from session three "adolescence": Computer intervention: Describe a special moment in your life as a teenager that you enjoyed a lot, e.g. a birthday party, or your first kiss. How did you feel in that moment? What did that mean to you? 
Face-to-face intervention: Did anyone make fun of you as a teenager or treat you disrespectful? Do you remember such a moment? What did that mean to you? How did you fell in that situation?

The questions were treated as suggestions; if the patient wished to add or delete something he or she was free to do so. Furthermore, the therapist was free to adapt and extend the questions based on the individual information the patient provided. The therapist could, for example, ask about current or past hobbies in two different ways based on the provided information. If the information suggested that a hobby might be a resource, i.e. a positive experience, one could ask: "I would like to invite you to tell me more about your hobby hiking! You told me it used to be fun to go hiking with your family?" If the information provided indicated that a hobby could probably no longer be carried out (e.g. due to loss of physical health), the therapist could rephrase: "I would invite you to tell me more about the challenges that you are currently facing. You told me that you are no longer able to go hiking? What happened and what does this mean to you?".

Moreover, each patient was encouraged to think in each session about one especially positive event that could be worked with in the computer part. In the face-toface part, the patient and the therapist focused on both negative and positive experiences, situations and memories in the biographical past, with the particular aim of restructuring negative ones. During the additional computer intervention, the patient was encouraged to filter positive experiences and to describe them in detail. Therefore, two depression modules of the "Butler system" (Botella, et al., 2009) were introduced. During the exercises in the "Virtual environment" module, the patient was encouraged to recall in detail a positive event and to carry out further exercises to induce positive mood as described in the introduction section. The therapist sat next to the patient and 
provided support in particular by finding positive events, which was often difficult for depressive patients. In the so called "Book of Life" module, the patient was encouraged to write down this positive situation and could add photos and music if desired. Similar to the generative document which is produced in "Dignity Therapy" (Chochinov et al.,2005), all participants received at the end of the final session a printed version of their text and photographs of the Book of Life that they had developed together with the therapist during their participationThey were encouraged to further adopt the developed strategies (e.g. restructuring negative thoughts, focusing on positive events in their lives, or talking to significant others about their emotions) to reduce negative mood.. There was no check afterwards whether the participants actually followed the suggestion.

\section{Data analysis}

SPSS 17.0 for Windows was used for all analyses. In preliminary analyses, we compared the intervention and the control group at baseline using $t$ and chi-square tests. To test hypothesis 1, analysis of covariance (ANCOVA) for repeated measures was carried out including a between-group factor (intervention vs. control group) and a within-group factor (pre-treatment vs. post-treatment). The main focus was basically on the group $\mathrm{x}$ time interaction effect. Further, mean scores at follow-up (after 3 months) were compared with pre-treatment mean scores (ANOVA). In addition, partial correlations (Pearson) were calculated to examine the relationship between the depression residual gain scores and the investigated variables.

To quantify the magnitude of differences between the two groups (intervention versus control), we used Cohen's $d$ as a measure of effect size. Cohen (1992) distinguished between small $(d=.20)$, medium $(d=.50)$ and large $(d=.80)$ effect sizes.

As no participants dropped out after beginning the intervention, we did not conduct intention-to-treat analysis. 


\section{Results}

Since a preliminary analysis of the sample characteristics revealed significant age differences between the two groups, we controlled for age in the following analysis. A dichotomous age variable was calculated by creating two groups of younger and older participants using median split (70 years).

We hypothesized that the life-review therapy might lead to a reduction in depressive symptoms and an increase in self-esteem, life satisfaction, well-being and integrative reminiscence, as well as a decrease in obsessive reminiscence. As shown in Table 2, results from the ANCOVA revealed a significant group (intervention vs. control group) $\mathrm{x}$ time (pre-treatment vs. post-treatment) interaction effect for depression (BDI-II, F 11.46, $p<.01, d=0.72$ ), indicating that the decrease in depressive symptoms in the intervention group was significantly larger than in the control group. Analysis did not reveal significant effects of age as control variable (F .95, $p>.05)$. Further, the depression score decreased significantly in the intervention group from pre-treatment to post-treatment compared to the control group (F 4.49, $p<.05)$ and from pre-treatment to the 3-month follow-up ( $\mathrm{F} 18.21, p<.01)$. Results indicate a large effect size from pre- to post-treatment $(d=1.13)$ and from pre-treatment to follow-up $(d=1.27)$. Figure 2 shows the course of depressive symptoms by group over time.

Further, Table 2 shows results from the ANCOVA concerning self-esteem (SES). The analysis revealed a significant but smaller group $\mathrm{x}$ time effect (F 4.21, $p<$ .05) compared to depression and did not reveal significant effects of age as control variable $(\mathrm{F} .34, p>.05)$. The effect in this case was small $(d=.19)$ and self-esteem was not found to increase significantly between pre-treatment and follow-up (F .19, $p>.05)$ but decreased to the baseline level. Moreover, results showed no significant effect of factor (self-esteem) from pre-treatment to post-treatment (F .29, $p>.05)$. 
Results from the ANCOVA concerning well-being (WHO-5) showed no significant interaction effect (F 2.69, $p>.05$ ), and no significant effect of factor (wellbeing) from pre- to post-treatment (F .01, $p>.05)$, , but a significant effect in the intervention group from pre- to 3-month follow-up (F 5.39, $p<.05, d=.70$ ). Similarly, there was no significant interaction effect (F .00, $p>.05)$ concerning the obsessive reminiscence (RQ), nor did this variable decrease significantly from pre- to posttreatment in the intervention group compared to the control group (F 2.24, $p>.05)$. However, the obsessive reminiscence (RQ) decreased significantly in the intervention group from pre-treatment to the 3-month follow-up (F 7.43, $p<.05, d=.93)$. Results concerning integrative reminiscence (RQ) did not show any significant interaction at post-treatment (F 2.69, $p>.05)$, effect of factor ( $\mathrm{F} .09, p>.05)$, or from pre-treatment to follow-up (F 4.61, $p>.05)$.

With regard to life satisfaction (LSIA), results did not indicate a significant interaction effect (F 1.10, $p>.05)$, but the results showed a significant effect of factor (F 6.52, $p<.05, d=.58$ ), indicating that the life satisfaction decreased significantly in the control group. Furthermore, analyses revealed a significant age influence (F 4.8, $p<$ .05). Results did not show a significant effect from pre-treatment to follow-up (F .37, $p$ $>.05)$.

Table 3 shows the correlations between the depression residual gain score (BDIII) and self-esteem (SES), well-being (WHO-5), life satisfaction (LSIA), integrative reminiscence (RQ) and obsessive reminiscence (RQ) at post-treatment (T1) and followup (T2). The depression residual gain score was calculated as the difference between the z-transformed BDI scores at post-treatment and baseline multiplied by the correlation between the two scores (Heinecke, Weise, \& Rief, 2010), i.e. lower values imply greater symptom reduction. Significant medium correlations were found between self-esteem 
(SES) and the depression residual gain score (BDI-II) at post-treatment $(\mathrm{r}=.70, p<$ .01 ), between the integrative reminiscence score (RQ) and the depression residual gain score $(\mathrm{BDI}-\mathrm{II})$ at post-treatment $(\mathrm{r}=-.48, p<.05)$, between the obsessive reminiscence score (RQ) and the depression residual gain score (BDI-II) at pre-treatment $(\mathrm{r}=-.55, p$ $<.05)$, and further, between the well-being score (WHO-5) and the depression residual gain score (BDI-II) at the 3-month follow-up $(\mathrm{r}=-.57, p<.05)$.

\section{Discussion}

The aim of this study was to investigate a structured and time-limited (six-week) lifereview therapy in a randomized controlled trial (waiting list) with depressive older adults in a face-to-face setting with additional use of two depression modules of the emental health "Butler system" (Botella, et al., 2009). To our knowledge, this is the first study to investigate a life-review therapy in this combined setting for depression in older adults.

First, we examined whether our intervention led to a reduction in depressive symptoms. Our results showed that the depressive symptoms decreased significantly over time until the 3-month follow-up in the intervention group compared to the control group. Analysis revealed medium to large effect sizes. These findings are in line with previous studies reporting that life-review therapy is an effective intervention to reduce depressive symptoms in older populations (Bohlmeijer, et al., 2003; Pot, et al., 2010; Serrano, et al., 2004).

Furthermore, the drop-out rate in our sample was low. Only one individual in the intervention group discontinued participation after signing the informed consent but before starting treatment. Three participants in the control group dropped out before the post-assessment and before starting the treatment. This is in line with Bohlmeijer et al. (2003), who reported relatively low drop-out rates in their meta-analysis. 
Furthermore, we investigated whether our intervention led to an increase in well-being and a decrease in obsessive reminiscence among the participants in the intervention group. Results indicate that this was not the case from pre- to posttreatment, but did occur from pre-treatment to follow-up. As mentioned above, none of the responders received psychotherapeutic treatment elsewhere during this time period, i.e. this result could be interpreted as a further intervention effect. Concurrently, the depression score decreased further from post-treatment to follow-up. This may indicate that the intervention caused further positive effects among the participants.. Pinquart and Forstmeier (2012) reported small effect sizes regarding positive well-being at posttreatment $(g=0.33)$ and follow-up $(g=0.32)$. One could speculate that after the end of treatment, the individuals in the current study probably continued to practice the strategies which they had developed during the therapeutic process, and were probably coping better with problems arising in their lives. As mentioned above, at the end of the final session, all participants received a printed version of their text and photographs of the Book of Life in order to further adopt the developed strategies.

We further found a significant negative correlation between well-being and the depression residual gain score at follow-up, indicating that a higher sense of well-being is related to greater symptom reduction. The residual gain score was calculated such that a negative sign indicated improvement, i.e. symptom reduction. Moreover, results showed that individuals with higher levels of obsessive reminiscence at baseline and integrative reminiscence at post-treatment benefited more from the intervention. Our hypothesis that integrative reminiscence might increase during treatment failed to reach significance. This finding is comparable to Pot et al. (2010), who did not find any significant changes in reminiscence types after a life-review intervention. Since 
different modes of reminiscence were not directly addressed in our life-review therapy the participants were only stimulated to use this kind of remembering in general.

Furthermore, results did not show a significant change in life satisfaction. One could speculate that life satisfaction might be recognized as a more stable construct, i.e. is personality linked (Ryff, 1989), which would be more challenging to change during a short-term (six-session) therapeutic intervention. One could speculate that the investigated treatment addressed more explicitly the reduction of depressive symptoms than the enhancement of life satisfaction. Analysis further indicated a significant but small interaction effect concerning self-esteem and showed that higher self-esteem scores at post-treatment were correlated with lower levels of decrease in depressive symptoms. As self-esteem probably shows high correlational stability and a negative concurrent correlation with depression, this result may indicate that individuals with lower mental health problems benefited less from the intervention.

The limitations of our study include a low sample size $(N=36)$ and a bias due to self-selection based on our recruitment advertising. $31 \%$ of the participants had previous experience of psychotherapy and indicated high interest and motivation. Further, we selected a rather homogeneous sample due to our strict exclusion criteria, e.g. comorbidity. It is well known that depression co-occurs with other disorders (Hautzinger, 2000). These facts may limit the generalizability of our results, and future research should focus more on comorbidity when investigating depression in elder populations.

A further limitation of our study was the inclusion of a waiting-list control group. Our results indicate that the intervention was an effective treatment to decrease depressive symptoms among depressive older adults. However, we cannot state that this intervention was as effective, or even more effective, than treatment as usual (e.g. 
standard CBT). Investigating this intervention in a randomized controlled setting with a treatment-as-usual control group could be a valuable next step in researching life review in this combined setting. As Pinquart and Forstmeier (2012) indicated, the effect size of reminiscence intervention drops significantly to about 0.4 when it is compared to an active control. Furthermore, a possible social interaction effect (e.g. interaction with another person, looking together at the text and photos) may have contributed to the comparably high effect sizes found in this short time intervention.

Considering these limitations, it is nevertheless noteworthy that we found significant medium-to-high effect sizes in a rather low sample, indicating that a lifereview therapy in this combined setting could be recommended for older adults aged 65 or over. To our knowledge, this is the first randomized controlled trial in this context, and therefore our study contributes to providing a better understanding of the effects of life-review therapy with computer supplements on depression among older adults, replicating previous findings on traditional face-to-face life-review interventions (see Pinquart \& Forstmeier, 2012).).

\section{References}

Bech, P. (1998). Quality of life in the psychiatric patient. London: Mosby-Wolfe.

Beck, A. T., Steer, R. A., \& Brown, G. K. (1996). Manual for the Beck Depression Inventory-II. San Antonio: TX: Psychological Corporation.

Beekman, A. T., Geerlings, S. W., Deeg, D. J., Smit, J. H., Schoevers, R. S., de Beurs, E., et al. (2002). The natural history of late-life depression: a 6-year prospective study in the community. Archives of General Psychiatry, 59, 605-611. http://dx.doi.org/10.1001/archpsyc.59.7.605 
Bohlmeijer, E., Roemer, M., Cuijpers, P. C., \& Smit, F. (2007). The effects of reminiscence on psychological well-being in older adults: A meta-analysis. Aging \& Mental Health, 11, 291-300. http://dx.doi.org/10.1080/13607860600963547

Bohlmeijer, E., Smit, F., \& Cuijpers, P. (2003). Effects of reminiscence and life review on late-life depression: a meta-analysis. International Journal of Geriatric Psychiatry, 18, 1088-1094. http://dx.doi.org/10.1002/gps.1018

Botella, C., Etchemendy, E., Castilla, D., Banos, R. M., Garcia-Palacios, A., Quero, S., et al. (2009). An e-health system for the elderly (Butler Project): a pilot study on acceptance and satisfaction. CyberPsychology and Behavior, 12, 255-262.

http://dx.doi.org/10.1089/cpb.2008.0325

Chiang, K. J., Lu, R. B., Chu, H., Chang, Y. C., \& Chou, K. R. (2008). Evaluation of the effect of a life review group program on self-esteem and life satisfaction in the elderly. International Journal of Geriatric Psychiatry, 23(1), 7-10. http://dx.doi.org/10.1002/gps.1824

Chochinov, H.M., Hack, T., Hassard, T., Kristjanson, L.J., McClement, S., \& Harlos, M. (2005). Dignity therapy: a novel psychotherapeutic intervention for patient near the end of life. Journal of Clinical Oncology, 23(24), 5520-5525. doi:10.1200/JCO.2005.08.391

Cohen, J. (1992). A power primer. Psychollogical Bulletin, 112, 155-159. doi:10.1037//0033-2909.112.1.155

Cole, M. G., \& Dendukuri, N. (2003). Risk factors for depression among elderly community subjects: a systematic review and meta-analysis. The American Journal of Psychiatry, 160, 1147-1156. http://dx.doi.org/10.1176/appi.ajp.160.6.1147 
Folstein, M. F., Folstein, S. E., \& McHugh, P. R. (1975). "Mini-mental state": A practical method for grading the cognitive state of patients for the clinician. Journal of Psychiatric Research, 12, 189-198.

Haight, B. K., \& Dias, J. K. (1992). Examining key variables in selected reminiscing modalities. International Psychogeriatrics, 4, 279-290.

Haight, B. K., \& Haight, B. S. (2007 ). The handbook of structured life review.

Baltimore: Health Professional Press, Inc.

Gray-Little, B., Williams, V.S.L. \& Hancock, T.D. (1997). An Item Response Theory Analysis of the Rosenberg Self-Esteem Scale. Personality and Social Psychology Bulletin, 23, 443-451. doi: 10.1177/0146167297235001

Hautzinger, M. (2000). Depression im Alter. Weinheim: Beltz/ PVU.

Hautzinger, M., Keller, F., \& Kühner, C. e. a. (2006). Das Beck Depressionsinventar II. Deutsche Bearbeitung und Handbuch zum BDI II. Frankfurt a. M.: Harcourt Test Services.

Heinecke, K., Weise, C., \& Rief, W. (2010). [Chronic tinnitus: which kind of patients benefit from an outpatient psychotherapy?]. Psychotherapie $\cdot$ Psychosomatik Medizinische Psychologie, 60, 271-278.

Maercker, A. (2002). Life-review technique in the treatment of PTSD in elderly patients: rationale and three single case studies. Journal of Clinical Geropsychology, 8 , 239-249. http://dx.doi.org/10.1023/A:1015952429199

Liang, J. (1984). Dimensions of the Life Satisfaction Index A: A Structural Formulation. The Journals of Gerontology, 39, 613-622. doi: 10.1093/geronj/39.5.613 Mayer, A.-K., Filipp, S.-H., \& Ferring, D. (1996a). Der Reminiszenzfragebogen: Skalenkonstruktion und teststatistische Überprüfung. Diagnostica, 42, 175-189. 
Oswald, W. D., \& Fleischmann, U. M. (Eds.). (1995). Nürnberger-Alters -Inventar (NAI). Manual. Göttingen: Hogrefe.

Parsons, T. D., \& Rizzo, A. A. (2008). Affective outcomes of virtual reality exposure therapy for anxiety and specific phobias: A meta-analysis. Journal of Behavior Therapy and Experimental Psychiatry, 39, 250-261.

http://dx.doi.org/10.1016/j.jbtep.2007.07.007

Pinquart, M., Duberstein, P.R. \& Lyness, J.M. (2007). Effects of psychotherapy and other behavioral interventions on clinically depressed older adults: A meta-analysis. Aging \& Mental Health, 11, 645-657. doi:10.1080/13607860701529635

Pinquart, M., \& Forstmeier, S. (2012). Effects of reminiscence interventions on psychological outcomes: A meta-analysis. Aging and Mental Health, 1-18, iFirst. http://dx.doi.org/10.1080/13607863.2011.651434

Pot, A. M., Bohlmeijer, E. T., Onrust, S., Melenhorst, A. S., Veerbeek, M., \& De Vries, W. (2010). The impact of life review on depression in older adults: a randomized controlled trial. International Psychogeriatrics, 22, 572-581.

http://dx.doi.org/10.1017/S104161020999175X

Preschl, B., Wagner, B., Forstmeier, S., \& Maercker, A. (2011). E-Health Interventions for Depression, Anxiety Disorders, Dementia and Other Disorders in Older Adults: A Review. Journal of CyberTherapy \& Rehabilitation, 4(3), 371-385.

Ryff, C. D. (1989). Happiness Is Everything, or Is It? - Explorations on the Meaning of Psychological Well-Being. Journal of Personality and Social Psychology, 57, 10691081. http://dx.doi.org/10.1037/0022-3514.57.6.1069

Serrano, J. P., Latorre, J. M., Gatz, M., \& Montanes, J. (2004). Life review therapy using autobiographical retrieval practice for older adults with depressive 
symptomatology. Psychology and Aging, 19, 270-277. http://dx.doi.org/10.1037/08827974.19.2.272

Webster, J. D. (1993). Construction and validation of the Reminiscence Function Scale. Joural of Gerontology: Psychological Sciences, 48, 256-262.

Webster, J. D., Bohlmeijer, E. T., \& Westerhof, G. J. (2010). Mapping the Future of Reminiscence: A Conceptual Guide for Research and Practice. Research on Aging, 32 (4), 527-564. doi:10.1177/0164027510364122

Wendt, H. (Ed.). (1979). Rosenberg Self-Esteem Scale - deutsche Fassung Rosenberg Self-Esteem Scale (Rosenberg, M., 1965) - German version/author. München: Pfeiffer.

Wernicke, T. F., Linden, M., Gilberg, R., \& Helmchen, H. (2000). Ranges of psychiatric morbidity in the old and the very old--results from the Berlin Aging Study (BASE). European Archives of Psychiatry and Clinical Neuroscience, 250, 111-119. http://dx.doi.org/10.1007/s004060070027

Westerhof, G. J., Bohlmeijer, E., \& Webster, J. D. (2010). Reminiscence and mental health : a review of recent progress in theory, research and interventions. Ageing \& Society 30, 697-721. doi:10.1017/S0144686X09990328

Wiendieck, G. (1970). Entwicklung einer Skala zur Messung der Lebenszufriedenheit im hoeheren Lebensalter. Zeitschrift für Gerontologie, 3, 215-224.

Wittchen, H.-U., Zaudig, M., \& Fydrich, T. (1997). Strukturiertes Klinisches Interview für DSM-IV. Göttingen: Hogrefe.

Wong, P. T. P., \& Watt, L. M. (1991). What Types of Reminiscence Are Associated with Successful Aging? Psychology and Aging, 6, 272-279.

http://dx.doi.org/10.1037/0882-7974.6.2.272 
Table 1: Sample characteristics.

Table 2: Means, standard deviations and effect sizes for depression and self-esteem by treatment and control group.

Table 3: Correlations of the SES (self-esteem), WHO-5 (wel-lbeing), LSIA (life satisfaction), integrative rem. (RQ) and obsessive rem. scores at pre- and post-treatment with the BDI residual gain score at post-treatment and follow-up in the intervention and control group.

Figure 1: Flowchart of participant progress.

Figure 2: Mean depression score over time.

\section{Acknowledgements}

The authors would like to thank Jürgen Raithel, who served as therapist in the study. During the work on her dissertation, Barbara Preschl was a pre-doctoral fellow of the International Max Planck Research School on the Life Course (LIFE, www.imprslife.mpg.de; participating institutions: MPI for Human Development, HumboldtUniversität zu Berlin, Freie Universität Berlin, University of Michigan, University of Virginia, University of Zurich). 


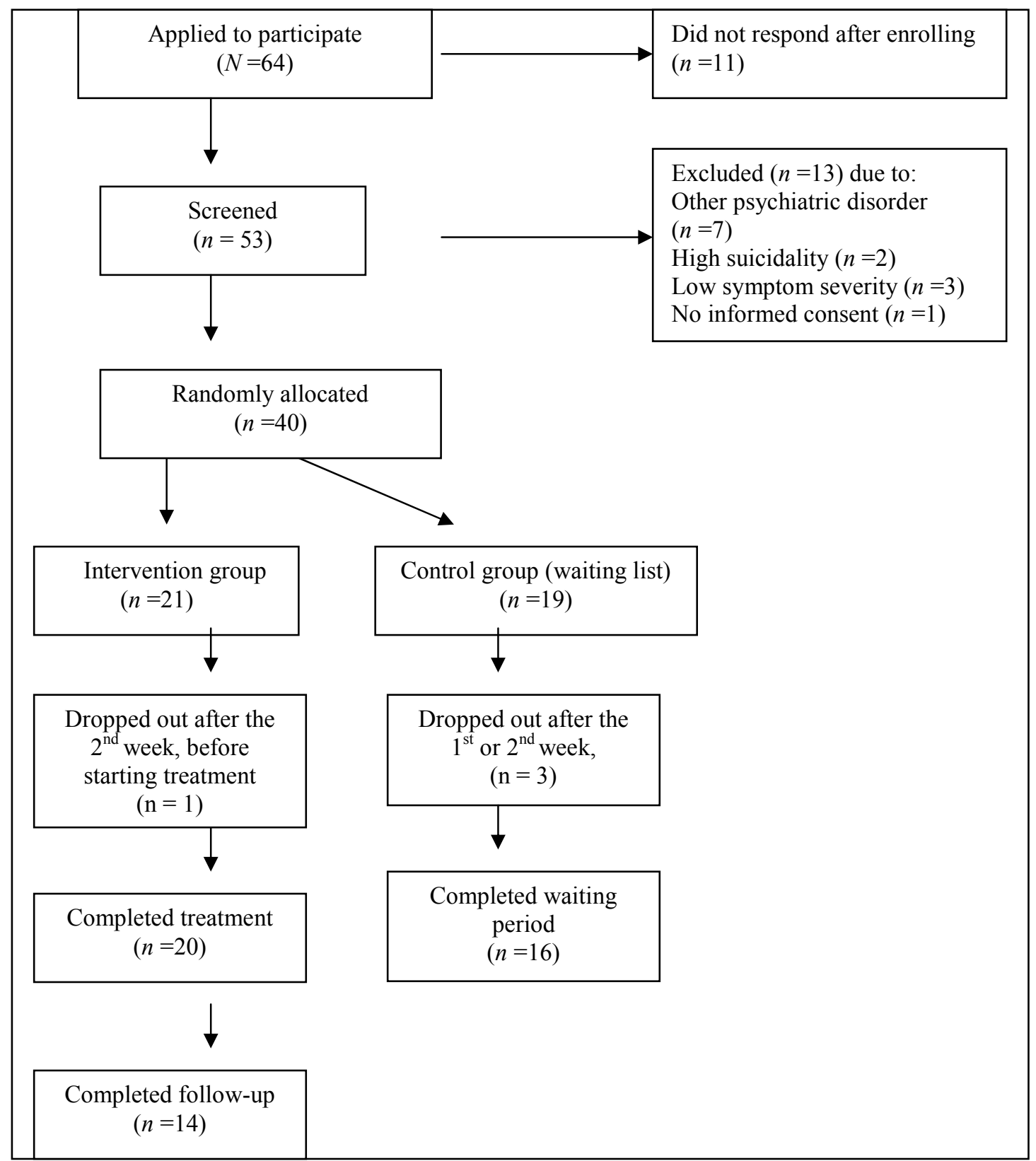

Figure 1: Flowchart of participant progress. 


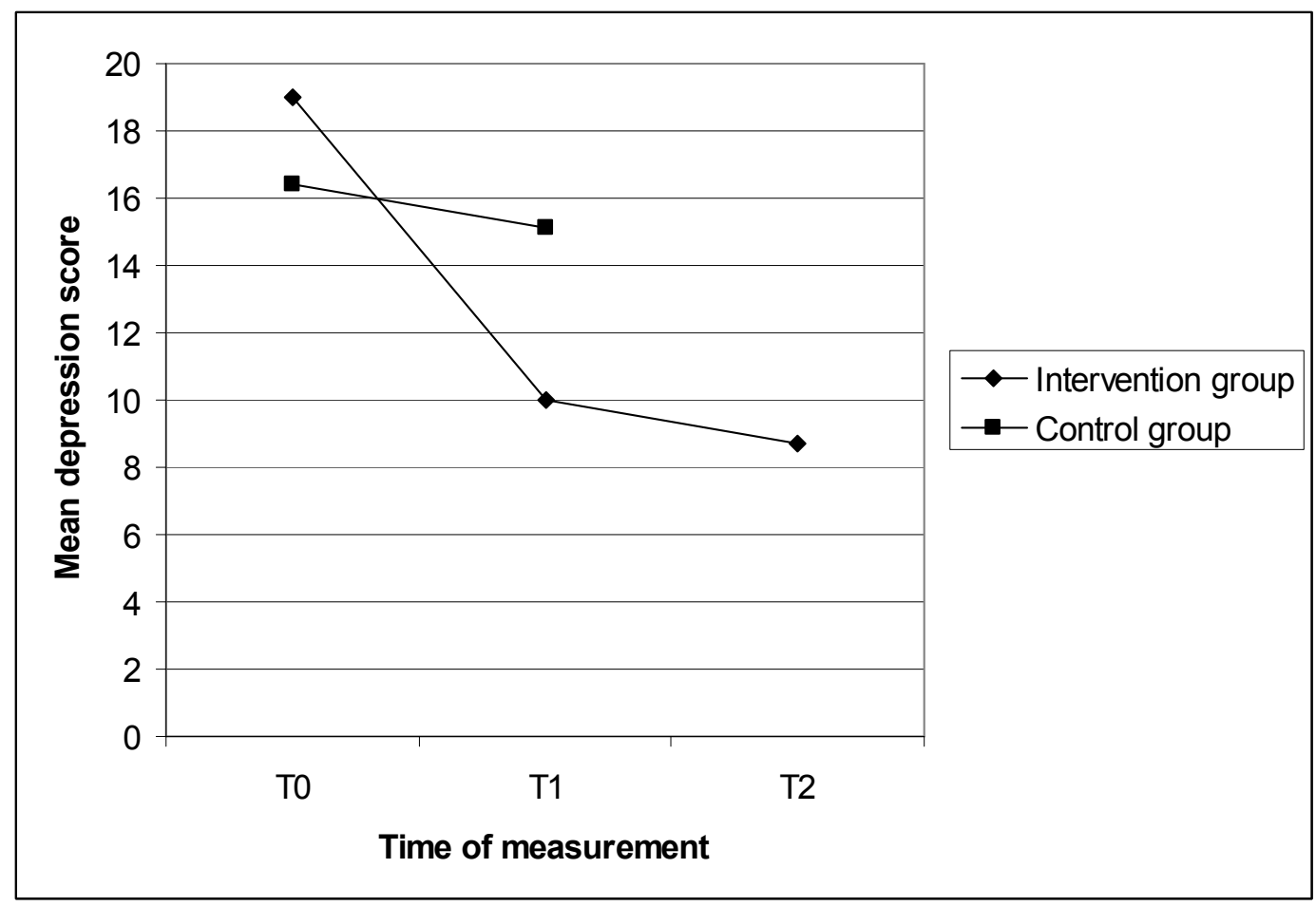

Figure 2: Mean depression score over time.

Note. Depression (Beck Depression Inventory, BDI-II) by group (intervention vs. control) and time of measurement $(n=36)$. 
Table 1: Sample characteristics.

\begin{tabular}{|c|c|c|c|c|}
\hline Characteristics & $\begin{array}{l}\text { Total sample } \\
(n=36)\end{array}$ & $\begin{array}{l}\text { Intervention } \\
\text { group } \\
(n=20)\end{array}$ & $\begin{array}{l}\text { Control group } \\
(n=16)\end{array}$ & $\begin{array}{l}\text { Group comparison } \\
\text { (df) }\end{array}$ \\
\hline Age, Median (SD) (in years) & $70.0(4.4)$ & $72.5(4.5)$ & $67.0(3.1)$ & $\chi^{2}(1)=5.36, p<.05$ \\
\hline Gender (female) & $24.0(66.7 \%)$ & $15.0(75 \%)$ & $9.0(56.3 \%)$ & $\chi^{2}(1)=.25, \mathrm{~ns}$ \\
\hline Marital status & & & & $\chi^{2}(3)=.7, \mathrm{~ns}$ \\
\hline Married/cohabiting & $19.0(52.8 \%)$ & $9.0(45 \%)$ & $10.0(62.5 \%)$ & \\
\hline Divorced & $9.0(25 \%)$ & $7.0(35 \%)$ & $2.0(12.5 \%)$ & \\
\hline Widowed & $8.0(22.2 \%)$ & $4.0(20 \%)$ & $4.0(25 \%)$ & \\
\hline Educational level & & & & $\chi^{2}(5)=.44, \mathrm{~ns}$ \\
\hline low & $11.0(30.5 \%)$ & $8.0(40 \%)$ & $3.0(18.8 \%)$ & \\
\hline medium & $17.0(47.2 \%)$ & $9.0(45 \%)$ & $8.0(50.1 \%)$ & \\
\hline high & $8.0(22.3 \%)$ & $3.0(15 \%)$ & $5.0(31.1 \%)$ & \\
\hline \multicolumn{5}{|l|}{ Employment status } \\
\hline Retired & $36.0(100 \%)$ & $20.0(100 \%)$ & $16.0(100 \%)$ & \\
\hline No antidepressants & $25.0(65.8 \%)$ & $15.0(71.4 \%)$ & $10.0(58.8 \%)$ & $\chi^{2}(1)=.42, \mathrm{~ns}$ \\
\hline Previous psychotherapy & $11(31.4 \%)$ & $6(31.6 \%)$ & $5(31.3 \%)$ & $\chi^{2}(2)=.54, \mathrm{~ns}$ \\
\hline $\begin{array}{l}\text { Depression (BDI-II) score at } \\
\text { baseline M (SD) }\end{array}$ & $17.6(6.3)$ & $19.0(6.6)$ & $16.5(5.6)$ & $\mathrm{t}(38)=.20$, n.s. \\
\hline $\begin{array}{l}\text { Self-esteem (SES) score at } \\
\text { baseline M (SD) }\end{array}$ & $22.1(4.8)$ & $21.4(5.4)$ & $23.0(3.9)$ & $\mathrm{t}(34)=.33$, n.s. \\
\hline $\begin{array}{l}\text { Well-being (WHO-5) score at } \\
\text { baseline M (SD) }\end{array}$ & $12.1(6.0)$ & $10.5(5.8)$ & $14.1(5.9)$ & $\mathrm{t}(34)=-1.84$, n.s. \\
\hline $\begin{array}{l}\text { Life satisfaction (LSIA) score at } \\
\text { baseline M (SD) }\end{array}$ & $32.5(3.8)$ & $31.4(3.5)$ & $34.0(3.8)$ & $\mathrm{t}(34)=-2.18, p<.05$ \\
\hline $\begin{array}{l}\text { Integrative Reminiscence (RQ) } \\
\text { score at baseline M (SD) }\end{array}$ & $9.9(1.9)$ & $9.8(1.5)$ & $10.2(2.3)$ & $\mathrm{t}(34)=-.62$, n.s. \\
\hline $\begin{array}{l}\text { Obsessive Reminiscence (RQ) } \\
\text { score at baseline M (SD) }\end{array}$ & $10.3(3.2)$ & $10.3(2.9)$ & $9.8(3.6)$ & $\mathrm{t}(34)=.72$, n.s. \\
\hline
\end{tabular}


Note. BDI-II = Beck Depression Inventory, SES = Rosenberg Self-Esteem Scale, WHO-5 = WHO-Five Wellbeing Index, LSIA = Life Satisfaction Index, RQ = Reminiscence Questionnaire. 
Table 2: Means, standard deviations and effect sizes for depression and self-esteem by treatment and control group.

Group x pre-post effect

\begin{tabular}{|c|c|c|c|c|c|c|c|}
\hline & Pre-test & Post-test & Follow-up & $\begin{array}{l}\text { Effect size } \\
\text { pre to post }\end{array}$ & $\mathrm{F}$ & $p$ & $\begin{array}{l}\text { Effect size } \\
\text { pre to 3- } \\
\text { month } \\
\text { follow-up }\end{array}$ \\
\hline \multicolumn{8}{|l|}{$\begin{array}{l}\text { Depression- } \\
\text { BDI-II }\end{array}$} \\
\hline Treatment & $19.0(6.6)$ & $10.0(6.3)$ & $8.7(4.8)$ & $1.13^{\mathrm{a}}$ & 11.46 & $<.01$ & $1.27^{\mathrm{a}}$ \\
\hline Control & $16.5(5.6)$ & $15.1(7.8)$ & - & .26 & & & - \\
\hline \multicolumn{8}{|l|}{$\begin{array}{l}\text { Self-esteem } \\
\text {-SES }\end{array}$} \\
\hline Treatment & $21.4(5.4)$ & $22.4(4.1)$ & $21.4(4.4)$ & .19 & 4.21 & $<.05$ & 0 \\
\hline Control & $23(3.9)$ & $21.6(4.4)$ & - & .49 & & & - \\
\hline \multicolumn{8}{|l|}{$\begin{array}{l}\text { Well-being } \\
\text { WHO-5 }\end{array}$} \\
\hline Treatment & $10.5(5.8)$ & $14.5(4.5)$ & $14.6(4.0)$ & .51 & 2.69 & $>.05$ & $.70^{\mathrm{a}}$ \\
\hline Control & $14.1(5.9)$ & $13.1(5.9)$ & - & .15 & & & - \\
\hline \multicolumn{8}{|l|}{$\begin{array}{c}\text { Life } \\
\text { satisfaction } \\
\text { LSIA }\end{array}$} \\
\hline Treatment & $31.4(3.5)$ & $31.6(3.8)$ & $31.4(3.0)$ & 0 & 1.11 & $>05$ & 0 \\
\hline Control & $34.0(3.8)$ & $\begin{array}{l}32.6 \\
(3.42)\end{array}$ & - & $.58^{\mathrm{a}}$ & & & - \\
\hline \multicolumn{8}{|l|}{$\begin{array}{l}\text { Integrative } \\
\text { Rem. } \\
\text { (RQ) }\end{array}$} \\
\hline Treatment & $9.8(1.5)$ & $10.3(1.5)$ & $10.6(1.9)$ & .23 & 2.69 & $>.05$ & .53 \\
\hline Control & $10.2(2.3)$ & $9.4(2.9)$ & - & & & & - \\
\hline \multicolumn{8}{|l|}{$\begin{array}{l}\text { Obsessive } \\
\text { Rem. } \\
\text { (RQ) }\end{array}$} \\
\hline Treatment & $10.6(2.9)$ & $10.0(1.7)$ & $8.6(2.8)$ & .31 & .00 & $>.05$ & $.93^{\mathrm{a}}$ \\
\hline Control & $9.8(3.6)$ & $9.8(3.6)$ & - & 0 & & & - \\
\hline
\end{tabular}

Note. Treatment group: $n=20$ ( $n=14$ at follow-up), control group: $n=16,{ }^{\text {a }}$ significant effect; BDI-II $=$ Beck Depression Inventory, SES = Rosenberg Self-Esteem Scale, WHO-5 = WHO-Five Well-being Index, LSIA = Life Satisfaction Index, RQ = Reminiscence Questionnaire. 
Table 3: Correlations of the SES (self-esteem), WHO-5 (wel-lbeing), LSIA (life satisfaction), integrative rem. (RQ) and obsessive rem. scores at pre- and post-treatment with the BDI residual gain score at post-treatment and follow-up in the intervention and control group.

\begin{tabular}{|c|c|c|c|c|c|}
\hline \multirow[t]{4}{*}{ Variable } & \multirow{2}{*}{\multicolumn{4}{|c|}{$\begin{array}{l}\text { BDI residual gain score at post-treatment } \\
\text { (covariate: age) }\end{array}$}} & \multirow{4}{*}{$\begin{array}{l}\text { BDI residual gain } \\
\text { score at follow-up } \\
\text { Intervention group } \\
\text { Follow-up }\end{array}$} \\
\hline & & & & & \\
\hline & \multicolumn{2}{|c|}{ Intervention group } & \multicolumn{2}{|c|}{ Control group } & \\
\hline & Pre & Post & Pre & Post & \\
\hline Self-Esteem (SES) & .44 & $.70 * *$ & -.10 & -.38 & -.10 \\
\hline \multicolumn{6}{|l|}{ score } \\
\hline Well-being (WHO-5) & -.14 & -.30 & .02 & -.45 & $-.57 *$ \\
\hline \multicolumn{6}{|l|}{ score } \\
\hline Life satisfaction & .09 & -.17 & .29 & -.07 & -.05 \\
\hline \multicolumn{6}{|l|}{ (LSIA) score } \\
\hline Integrative Rem. & -.22 & $-.48 *$ & .14 & .10 & -.42 \\
\hline \multicolumn{6}{|l|}{ (RQ) score } \\
\hline Obsessive Rem. (RQ) & $-.55 *$ & -.38 & .24 & .38 & .02 \\
\hline
\end{tabular}

Note. BDI-II $=$ Beck Depression Inventory, SES $=$ Rosenberg Self-Esteem Scale, WHO-5 = WHO-Five Wellbeing Index, LSIA $=$ Life Satisfaction Index, $\mathrm{RQ}=$ Reminiscence Questionnaire; $* p<.05 . * * p<.01$. 